This is a postprint of an article that was published in the book:

"Constructions and Frames, Vol. 2(2010), no 2, pp. 158-184."

The published article is under copyright of Benjamins. The publisher should be contacted for permission to re-use or reprint the material in any form.

\title{
A constructional account of genre-based argument omissions ${ }^{\star}$
}

\author{
Josef Ruppenhofer and Laura A. Michaelis \\ Universität des Saarlandes / University of Colorado at Boulder
}

\begin{abstract}
Authors like Fillmore 1986 and Goldberg 2006 have made a strong case for regarding argument omission in English as a lexical and construction-based affordance rather than one based on general semantico-pragmatic constraints. They do not, however, address the question of how grammatical restrictions on null complementation might interact with broader narrative conventions, in particular those of genre. In this paper, we attempt to remedy this oversight by presenting a comprehensive overview of genre-based argument omissions and offering a construction-based analysis of genre-based omission conventions. We consider five genre-based omission types: instructional imperatives (Culy 1996, Bender 1999), labelese, diary style (Haegeman 1990), match reports (Ruppenhofer 2004) and quotative clauses. We show that these omission types share important traits; all, for example, have anaphoric rather than indefinite construals. We also show, however, that the omission types differ from each other in idiosyncratic ways. We then address several interrelated representational problems posed by the grammatical treatment of genre-based omissions. For example, the constructions that represent genre-based omission conventions must interact with the lexical entries of verbs, many of which do not generally permit omitted arguments. Accordingly, we offer constructional analyses of genre-based omissions that allow constructions to override lexical valence constraints.
\end{abstract}

Keywords: genre, argument structure, null complementation, derivation, Sign-Based Construction Grammar, instructional imperatives, diary omission, labelese, match reports, quotative clauses

\footnotetext{
* The work of the first author was funded in part by the German Research Foundation DFG under grant PI 154/9-3. For discussion and constructive criticism, we are indebted to Charles Fillmore, Adele Goldberg and two anonymous reviewers.
} 


\section{Introduction}

In analyses of null-complement phenomena, pragmatic factors play an important role. For example, Fillmore (1986) distinguishes two major types of null complements, definite and indefinite, based on the potential for a discourse antecedent, and Goldberg (2006: Chapter 9) uses the discourse prominence of participants to explain why constructions like the English experiential perfect license argument omissions that are not allowable in episodic contexts (e.g., She has never failed to impress $\varnothing$ ). But the primary aim of works in this tradition has been to establish that restrictions on argument omission, despite invoking contextual conditions, are grammatical constraints rather than general semantico-pragmatic ones. This case is made based on both lexical idiosyncrasies (e.g. the verb eat can omit its patient argument while its near-synonym devour cannot) and construction-specific argument-omission affordances (e.g., the theme argument of a transfer verb like load cannot be omitted except when it would be realized as an oblique expression, as in She loaded the truck $\varnothing$ ). In other words, it is a set of lexemes and constructions, rather than, say, norms of conversational practice or inference, that determine whether a given argument can be omitted and, if so, what its construal type (anaphoric or indefinite) will be. On this model, pragmatic conditions like low discourse prominence are, at least in English, necessary rather than sufficient conditions on non-realization of arguments; the specific construction determines whether null realization can actually occur. For example, the accomplishment verb arrest prohibits zero realization of the 'detainee' argument in episodic contexts, as in (1), but allows it in generic-habitual contexts, as in (2), an example retrieved from Google:

(1) ${ }^{\star}$ The cops arrested $\varnothing$ last night.

(2) Sure the cops arrest $\varnothing$ when they can, but it's always in small amounts.

The problem, as we see it, is that authors in this tradition have not looked beyond sentential context to address the role of narrative context, and in particular genre, in the creation of argument-omission affordances. This is not surprising: genres are not language objects like words or phrases, and therefore it is unclear how constructions can be used to represent genres. But genre and argument omission are closely connected conventions - so much so that speakers and writers can often evoke a rich genre by simply omitting the appropriate argument in a predication. For example, the title of Cynthia P. Lawrence's 2002 novel, Chill Ø before Serving Ø: A Mystery Novel for Food Lovers, ${ }^{1}$ evokes the Instructional Imperative

1. While both chill and serve are missing their second arguments in this example, the missing argument of the latter verb represents a parasitic gap rather than a case of definite null instantiation. 
genre by omitting the object of the imperative verb chill, while one can detect the diary genre in the subject-less predications of the Beatles' song "A Day in the Life": $\varnothing$ woke up, $\varnothing$ got out of bed/ $\varnothing$ dragged a comb across my head/ Ø found my way downstairs and Ø had a cup.

In this paper, we attempt to connect the study of genre to that of null complementation by identifying the dimensions of genre that are relevant to the use and interpretation of null arguments. Our data are drawn from several sources: the British National Corpus (Burnard 1995), a balanced 100-million word corpus of written and spoken English; the diaries of Virginia Woolf (Bell 1978-1984); and various internet search engines. We will make use of constructed examples, as indicated, to illustrate grammaticality judgments.

We will focus on five genre-based omission types that illustrate the range of phenomena a model of context-based argument omission must encompass: instructional imperatives, as in (3) (Culy 1996, Bender 1999), label statements or labelese, as in (4), diary style, as in (5) (Haegeman 1990), match reports, as in (6), and judgment-expressing quotative verbs whose evaluee argument is identified with the addressee (7) (Ruppenhofer 2004):

(3) Sweet Lassi Ingredients:

1 Serving Plain yogurt

1 cup Sugar

2 tablespoons Ice Cubes

Method: Blend all the ingredients in an electric blender. Serve $\varnothing$ cold.

(4) $\varnothing$ Contains alcohol.

(5) $\varnothing$ read Michelet; $\varnothing$ wrote to Desmond about his poetess; L. out at Fabians; $\varnothing$ played gramophone; ...

(6) He hammered $\varnothing$ wide of Gary Walsh's exposed net.

(7) Nice work, boys, she praised $\varnothing$ with a light smile.

With the exception of match reports, these are exclusively written genres. Nonetheless, they exhibit considerable variety: some could properly be called literary conventions (diary and quotative omissions) and some clearly could not (labelese). Our bottom line, however, is that all can be described as constraints on argument structure, as the relevant conventions target specific semantic and grammatical roles of verbs. We will attempt to capture the effect of genre on argument omission using constructions that license null complements, and that are conventionally associated with certain contexts of use. In our grammatical analysis, couched in the framework of Sign-Based Construction Grammar (Sag 2007, 2010), we will use 
the CONTEXT feature to represent the link between a given construction and its contexts of use.

The remainder of this paper will be structured as follows. In Section 2, we will establish that genre-based omissions are in fact constructional omissions and discuss the conception of genre to be assumed in this paper. In Section 3, we will discuss the nature of the connection between genre and argument omission. In Section 4, we will discuss commonalities among the five genre-based omission patterns, as well as evidence that they are distinct phenomena, requiring distinct constructional analyses. Section 5 will outline a construction-based analysis of genre-based omission. In Section 6 we will offer concluding remarks.

\section{Genre-based omissions are constructional}

In this section we will situate the five genre-sensitive omission types in the larger context of argument omissions. Following Fillmore (1986), we distinguish lexically licensed omissions from constructional ones. In the former case, the argumentomission affordance is licensed by a particular predicator, and nearly synonymous predicators may differ in omissibility of a given semantic role, as shown by (8).

(8) I understand (what you're saying).

(9) I grasp * (what you're saying).

In the latter case, it is the particular construction that determines the omissibility of a given argument, in a given syntactic role. For instance, $b y$-phrase agents in passive predications are omissible regardless of the lexical identity of the passiveform verb (10). Likewise, all imperatives can omit their subjects, as in (11).

(10) The arrow was found $\varnothing$ near what has been identified as a prehistoric hunting site.

(11) Tevez: $\varnothing$ Show me the money! [headline]

There are also constructions that not only allow but also enforce argument omission. For instance, the Way-construction (Goldberg 1995: Chapter 9), and some types of resultative constructions (Boas 2003), require object omission under an existential interpretation, as illustrated by the constructed examples in (12)-(13):

(12) She knitted (* ${ }^{*}$ sweaters) her way across the Atlantic.

(13) I just can't seem to drink (*alcohol) you off my mind. 
In (12), the 'product' argument of knit is obligatorily null instantiated, as the Wayconstruction requires the verb's second argument to be a definite NP headed by way. In (13), the 'draft' argument of drink is obligatorily null instantiated because the Caused Motion construction requires the verb's second argument to be a theme argument.

The kinds of argument omissions that we are concerned with here are constructional omissions that are strongly associated with particular genres. While lexical items also have unequal distributions across various genres, we will not treat lexical omissions as genre-based omissions on that basis. For an omission to be genre-based, we require that the construction at issue allow the omission only in some of the genres that it occurs in, or that the construction itself be limited to particular genres, if it always allows omission. Thus, for example, the omissibility of the subject of an imperative is not genre-based, as imperatives have this affordance regardless of genre. By contrast, in ordinary, non-instructional imperatives like (14), the object must be overt.

(14) Take ${ }^{\star}$ (the money) and run.

Instructional imperatives that allow omission of the object occur in genres like recipes, as in (3) above, or product user manuals, as in (15):

(15) Check motor protection filter every time you change the paper filter bag. Replace $\varnothing$ by a new one if it is very dirty.

Note further that genres do not license omissions independently of constructions. A comparison of (16) with (3) above shows that occurrence in an instructional context is insufficient to license object omission. Instead, the target object must be the object of an imperative-form predication (i.e., one with a null addressee argument):

(16) What you want to do is this. You take the ingredients and blend *(them) for 10 minutes. Then you pour the mass into a pan and fry ${ }^{\star}$ (it) until it's golden brown. Then you take ${ }^{\star}($ it $)$ out of the pan and let ${ }^{\star}$ (it) cool for an hour. Finally, you serve ${ }^{\star}($ it $)$ cold.

Thus, we cannot capture object omission in instructional imperatives by deriving a genre-dependent lexical entry that would then be free to combine with all the usual constructions, as this would overgenerate. An alternative solution might be to say that (16) does not belong to an instructional context in the same way that (15) does, but rather exemplifies some other genre. The solution is unsatisfactory because it is circular: argument omission is assumed to be licensed by the genre and the genre is recognized based on argument omission. Instead, we recognize an 
omission construction that is formally constrained (the verb must be imperative) and associated with a specific type of discourse.

What conception of genre is relevant to the description of genre-sensitive constructions? As works like Biber (1995) and Biber et al. (2007) show, the linguistically relevant conception of genre is in many respects similar to that of register: a set of situational variables (like the relative social statuses of the interlocutors, the degree of formality of the communication and the communicative channel used) that are linked to variables of linguistic form (like speech rate, use of contraction and the presence of affective vocabulary). However, the conception of genre used by Biber and colleagues is a good deal more inductive than the one assumed here: it is based on the comparative analysis of large-scale patterns in various corpora. Further, the Biber conception of genre has far greater dimensionality: genre labels are merely shorthands for large clusters of linguistic features. Finally, the goal of Biber's enterprise, as Biber et al. (2007:261) point out, is "the linguistic analysis of texts, genres/registers and text types rather than analysis of individual linguistic constructions".

In the current study, we focus on a single linguistic variable (presence or absence of a null complementation affordance) and seek to describe null-complement-licensing constructions that are conventionally associated with certain text types, rather than to posit or defend the existence of certain text types on linguistic grounds. The genres or text types that we see as relevant to the description of null complementation affordances have more in common with the speech genres of Bakhtin (1986) than they do with Biber-style feature complexes. Like the speech genres described by Bakhtin, the genres assumed in the current analysis are situationally restricted speech patterns that are culturally important, manipulable, combinable and familiar enough to form the basis of acts of humorous pretense. On this understanding, a genre is not simply the coalescence of certain contextual and linguistic features but rather a socially relevant linguistic routine that one enacts through the selection of words and constructions that evoke the genre.

\section{Genres and omissions}

We begin with six general observations about the connection between genres and argument omission. The first is that genres enable omissions that the predicator or construction would not otherwise permit, rather than blocking omissions that are possible outside of the genre. For instance, while in most written genres of English, subjects cannot be omitted, diary style allows subject omissions. Similarly, while direct objects are generally omissible in episodic contexts only when licensed by particular predicators (e.g., the activity verbs eat, drink and read), certain genres 
license object omissions that are otherwise permitted only in generic-habitual contexts. Comparison of (17) and (18) shows, for example, that objects in resultative constructions are omissible in match reports but not outside that genre. Likewise, comparison of (19) and (20) shows that objects modified by depictive secondary predicates are omissible in Instructional Imperative contexts but not generally otherwise:

(17) ${ }^{*}$ He threw $\varnothing$ into the bin.

(18) Paramatti put the ball back into the box and Panadic headed $\varnothing$ into the net.

(19) ${ }^{*}$ They instructed us to eat $\varnothing$ raw.

(20) Phil's Cherry Pie: cool Ø briefly, then eat $\varnothing$ warm.

The ungrammaticality of (19) seems particularly puzzling when one considers that eat is well known to permit a covert second argument. The fact that it does not in (19) appears to reflect a constraint on secondary predication: the arguments of secondary predicates are anaphoric, and covert arguments of activity verbs like eat are existentially construed, at least outside of the instruction-giving genre. This point leads to our second observation: genre-based omissions require anaphoric interpretation. That is, the omitted arguments in question are entities that are not only mutually identifiable to speaker and hearer but also a current joint focus of attention, e.g., the food item being manipulated in the instruction-giving genre, the product in the labelese genre, the ball in the match-report genre. As a consequence, null arguments in such contexts are replaceable by pronouns (e.g., Cool it briefly in (20)). Genre-based omissions are thus distinct from other constructionally licensed omissions, including those found in habitual-generic predications:

(21) We used to discuss $\varnothing$ and discuss $\varnothing$ until we were down to, say, two goalkeepers.

In the habitual context of (21), the verb discuss licenses a covert object that it does not otherwise permit (cf. We discussed ${ }^{*}$ (things) last night). Crucially, however, this covert object has an existential rather than anaphoric construal: it is replaceable by an indefinite pronoun like something or a lexical NP like our opinions, but not by a definite pronoun like it or them. Similar observations apply to the passive construction, which licenses a covert oblique argument denoting an agent. This covert argument may have either an anaphoric construal, as in (22), or an existential construal, as in (23):

(22) I indicated that his message had been received (by me).

(23) My purse was stolen (by someone). 
Thus, constructionally licensed null complements are not intrinsically anaphoric. ${ }^{2}$ Why then do genre-based omissions entail anaphoric construal? Perhaps because recoverability of the missing argument requires recourse to cultural stereotypes, in the same way that certain (nonanaphoric) lexically licensed omissions do:

(24) I love to read (magazines and books).

(25) She drinks (alcohol).

(26) He smokes (tobacco).

Just as reading, drinking and smoking conventionally involve certain types of participants, so recipes, product labels and match reports invoke a specific set of conventional participants (an edible substance, a product, a ball, etc.). This explanation does not go through, however, because the missing arguments in (24)-(26) are not in fact anaphoric: none are replaceable, for example, by the definite pronoun it. Genre-sensitive argument omissions are anaphoric because the relevant genres presuppose the salience of certain entities. This is true not only of indexical genres like labelese, which feature inherently self-referential predications, but also of referent-tracking genres like diary style and instructional imperatives.

Third, we note that as with all other types of lexical and constructional omissions, genre-based omissions are never obligatory. For example, it is not ungrammatical to overtly express an object in an instructional imperative. However, the practice of object omission in recipes is common: Culy (1996) reports that about $40 \%$ of all objects are omitted in a corpus of contemporary recipes. Both halves of the Gricean quantity maxim would seem to encourage speakers to exploit the null complementation opportunities offered by genres: while effort conservation favors argument omission, informativeness favors using linguistic conventions (like argument omission) that signal what text type is in play. The claim that omission serves to invoke text type accords with Bender's (1999) observation that genres are not prior to, and independent of the omission types, but rather are constituted in part by the practice of omission along with other characteristic lexico-grammatical choices.

Fourth, we assume that texts can exemplify more than one genre. This can happen in two ways. On the one hand, genres have properties that can be captured by a multiple-inheritance hierarchy (in terms of Sag 2007, 2010). For example, passages like (5) above exemplify the diary genre while also exemplifying the narrative-discourse genre, in which a sequence of sentences is understood to represent a real-world event sequence (Dowty 1986). On the other hand, one

2. There is, however, at least one constructionally licensed omission that is intrinsically anaphoric: imperative omission, to be discussed in Section 5. 
genre can be embedded in another in a given text. For instance, if we find a recipe included in a diary entry, then we can explain any missing objects in the recipe based on its instructional discourse type, rather than including diaries in the class of instructional texts or vice versa. Of course, in some cases it may be difficult to determine whether a part of a text exemplifies a separate genre or simply falls within the (wide) range of variation of the text's main genre.

Fifth, there are probably more genre-based omission constructions than those described in this paper. For example, medical records may feature argument omissions specific to that text type. Similarly, our description of match reports is based primarily on football/soccer match reports; it is conceivable that in reports from other sports, such as basketball or baseball, slightly different constraints can be observed. ${ }^{3}$ Conversely, we note that argument omissions are not the only grammatical hallmarks of genres. For example, while count nouns in English generally require determiners, they appear without determiners in genres including newspaper headlines, as in (27), and exercise instructions, as in (28):

(27) Sewage plant worker finds arm in plant.

(28) So, the grips is such: left hand grabs arm, right hand under arm, traps the shoulder.

Finally, as in other cases of argument omission, the constraints on genre-based omission must refer not only to semantic roles, both general and frame-specific (e.g., agent, theme, edible substance), but also to grammatical functions like subject and object. For example, the labelese predication in (29) could not be converted to that in (30):

(29) $\varnothing$ packaged in a facility that also processes nuts.

(30) ${ }^{\star} A$ facility that processes nuts packaged $\varnothing$.

Labelese not only requires the covert argument to refer to the labeled product but also restricts that argument to the grammatical function of subject. Similar observations can be made for other genre-based omissions. The lesson here is that argument-omission constraints are constraints on linking rules, i.e., argumentstructure constructions in the sense of Goldberg $(1995,2006)$.

3. Unfortunately, many of the genres that are of interest to us, such as product labels, product manuals, medical records, match reports, etc., are absent or are rare in commonly used corpora. 


\section{Commonalities and differences among the constructions}

\subsection{Reference to prominent participant}

The discourse status of the omitted referent varies across omission types. Three of the five constructions restrict omission to a globally prominent referent: the ingredients of the recipe or other manipulated item, the contents of the container bearing the label, or the ball as the object of play. In the case of diaries, however, the author is only the most typical reconstruction of a null subject argument; other referents are also subject to omission, if they are topical in a stretch of discourse. Consider example (31), taken from the diaries of Virginia Woolf:

(31) The West boy [Harry] writes he's hunting his battalion-no clothes- $\emptyset$ won't go back, he says; but $\varnothing$ gives no word of his wounds. Some say hes [sic] the only survivor of his regiment. $\varnothing$ Landed in a sailing boat at Ramsgate.

It would, however, be a mistake to identify topichood, or discourse prominence, with importance. Topichood is simply a function of an argument's degree of predictability in a given predication or series of predications (Lambrecht and Michaelis 1998). For example, the evocation of Harry in the diary passage in (31), along with the activation of the battle frame and frequent pronominal references (as in, e.g., the parenthetical he says) ensure recoverability of this referent in null-subject predications like $\varnothing$ landed in a sailing boat at Ramsgate. Thus, we take an entity to be topical if it can readily be reconstructed as the filler of a given argument position. By contrast, we take an entity to be important in a given text only if it has ongoing relevance to readers, and readers can infer qualities of the entity from the text. In match reports, for example, the players are important while the ball is not: readers do not typically make inferences about the ball based on the events of the match, so one ball is as good as another for the purpose of match reporting. There is, however, only one object of play, while there are multiple players. Thus, the ball is highly topical: it is the only entity that could occupy the argument position it does in a given match-report predication, e.g., object of the verb headed in (18). One could therefore refer to the object of play by means of a pronoun without creating ambiguity. Accordingly, we might view null expression as the match-report analog to anaphoric reference.

We can contrast the instructional imperative, labelese and match-report omission types with lexical omissions such as (32), where there is no requirement that the referent of the zero argument have global discourse prominence. In (32), the writer lists several presumably unfamiliar food items and compares their properties. The 'tornado potato' is neither unique (as a token of its type) nor important 
(it is not mentioned again after its introduction); however, the reader must reconstruct it as the standard-of-comparison argument of similar:

(32) The tornado potato is an entire potato, cut into a spiral, flash fried and put on a stick. The Texas tater dog is similar $\varnothing$, only the potato is coiled around a German sausage. There's also the fry dog, which is a hot dog on a stick encrusted with french fries.

Lexically licensed omission does, however, provide a good model for the omission of addressees/evaluees in quotatives. The verbs that appear in this construction (e.g., scorn, criticize, praise) all appear to belong to three frames related by inheritance in the FrameNet hierarchy (Baker et al. 1998), namely Judgment, Judgment_ communication, and Judgment_direct_address. Further, the omitted referent can be anything that meets the predicate's selectional restrictions and is accessible in the discourse. It need not be, for instance, the main character or the character whose point of view is currently taken, as suggested by (33):

(33) "Come in!" Pomona Sprout had her back to the student entering the room. She was in the middle of watering one of her beautiful Fainting Lilies, which trembled at the vibration of the door closing, prostrating itself along the window sill. "Stop being melodramatic. It's only one of my fifth year students," she scolded Ø, settling herself in her comfortable, if rather mucky chair.

In (33), the scolder (she) is the main character (Pomona Sprout) whose point of view is taken throughout the text, while the recipient of the scolding is the potted lily that she happens to be watering when the story opens. What this suggests is that the omitted addressee/evaluee need only be a participant in the conversation depicted; it need not represent an important or unique entity.

Among the omission types that target prominent participants, we observe that continuous local topicality is not necessary for omission to be acceptable. Example (5) from Virginia Woolf's diary, repeated here as (34), illustrates that other referents, in this case the writer's husband Leonard, can intervene between co-referent omissions of the discourse topic.

(34) Ø read Michelet; Ø wrote to Desmond about his poetess; L. out at Fabians; $\varnothing$ played gramophone; ...

Similar observations apply to the recipe in (3) above: unless we take the omitted object of serve to be co-referential with the recipe's title, there is no prior mention in the body of the recipe of the final product. Finally, in instructional imperatives on labels we sometimes find that the referents in a series of omissions are actually different. For instance, in example (35), the omitted objects of shampooing and 
comb must be reconstructed as either the user or the user's hair, but the object of apply is the dab of balm:

(35) After shampooing $\varnothing$, squeeze a dab of balm into the palm, comb $\varnothing$ and apply $\varnothing$ evenly throughout wet hair starting at the roots down to the ends.

Examples like (35) suggest that argument omissions in label instructions exploit not only the identifiability of the container's product but also interrelations among participants in the frame to which the product belongs, e.g., the substance and its intended site of application in the case of the cosmetic frame.

\subsection{Canonicity}

A further difference among genres is that some genre-sensitive argument-omission constructions can combine with any predicate, whereas others require predicates to denote actions or properties that are canonical in the genre. For instance, in diary style, the subject of any predicate is subject to omission. Similarly, in the case of object omission in the quotative construction, no particular judgment-expressing reporting verbs can be considered canonical. By contrast, labelese predications are limited to those that describe the provenance, constitution, qualities or efficacy of the product, e.g., ø contains no hydrogenated oils, ø creates visibly fuller, thicker hair. Labelese predications like that in (36), an altered version of a statement found on a box of quinoa, are not attested:

(36) ${ }^{*} \varnothing$ has flourished in cultivation for over 5,000 years.

Match reports may include non-canonical events involving the ball. For instance, players sometimes step on the ball, kiss or rub it for good luck, hide it under their shirts, etc. However, we find omissions only with predicates denoting canonical aspects of the game such as taking various kinds of kicks or headers. Omissions like that in (37) are not attested:

(37) Before he took that free kick he kissed ${ }^{\star}$ (the ball) for luck.

In other words, superstitious gestures, while familiar to sports fans, are not a canonical part of any game. Along these same lines, one can also note that in sports broadcasts the use of the simple present tense is restricted to canonical actions on the field, as pointed out by Charles Fillmore (p.c.). Thus, it would be odd to continue the baseball report in (38) with the report in (39).

(38) Now that the middle innings are over with, the managers are bringing in their heavy artillery from the bullpen. Right-hander Mike Timlin relieves Arroyo and sets the Cardinals down in order. Millar has had a rough night 
in the field but he makes a nice pick on an errant Timlin throw for the third out. Kelly Clarkson comes in and finishes up her God Bless America rendition quicker than you can say, "Irish Tenor."

(39) A fan jumps over the outfield wall, strips, and runs naked across the field.

In light of the tense restriction observed in (38)-(39), it is not surprising that omissions may be restricted to predications denoting canonical properties and relations within the genre.

\subsection{Grammar}

As discussed in Section 3, genre-based omission conventions incorporate restrictions on grammatical function: instructional imperatives, match reports and the quotative construction allow for the omission of objects, while diary style and labelese statements allow only for the omission of subjects. Accordingly, attempts to omit objects in diary style or in descriptive statements on labels are not acceptable:

(40) $\varnothing$ wonder why my neighbor hates ${ }^{*}(\mathrm{me})$ so.

(41) Storing in dark room keeps ${ }^{*}$ (contents) fresh.

Note that the omission in (42) below, though found on a product label, is an instance of the instructional imperative construction rather than instantiating a more general construction that licenses the omission of both subjects and objects in the labelese genre:

(42) Store $\varnothing$ away from direct sunlight.

Once we move beyond grammatical function, a rigorous characterization of syntactic restrictions on genre-based omission remains elusive. Consider, for instance, the issue of whether the omission patterns in question occur in main clauses only or also in embedded clauses. As Haegeman (1991) observes, diary subjects are omissible only in main clauses. Compare the attested (43) with the doctored version on (44), where a subject omission occurs under embedding.

(43) Angelica there: I diagnose strain; a little defiance, restlessness. And $\varnothing$ feel in Q. something heavy, mature, depressed.

(44) Angelica there: I diagnose strain; a little defiance, restlessness. \#And I think $\varnothing$ feel in Q. something heavy, mature, depressed.

By contrast, in match-report omission can occur in embedded clauses: 
(45) Williams prevented worse damage with excellent saves from Ellington and Roberts, but Wigan missed a good chance when Graham hammered Ø wide after non-stop midfielder Jimmy Bullard set up the shooting chance.

In the case of instructional imperatives, failure to embed follows from imperative status. For descriptive statements on labels and quotative omissions, we cannot judge readily on the basis of attested data whether omissions can occur under embedding. Sentences like (46) and (47) were not to be found:

(46) The social worker's report states that the parent then scolded ?(the child), "Don't cry so much".

(47) HaPi guarantees that ${ }^{\star}$ (this product) has no material defects.

Based on introspection, we assume that embedding of quotatives like (46) is possible in principle, while embedding of label statements such as (47) is not.

How do the genre-based omission conventions interact with non-declarative syntax? ${ }^{4}$ Haegeman 1991 argues that missing subjects in diary style cannot occur in constructions containing a preclausal topical or focal referent (48), or in auxiliary-initial predications (49): ${ }^{5}$

(48) This book, *(I) did not approve of (cf. Haegeman 1991, (13a/b)

(49) When will *(I) be able to meet him? (op.cit., (14b))

For label statements, no useful attested data were found bearing on compatibility with fronting constructions. We will thus assume that subject omission in label statements does not occur in predications with clause-initial constituents. What of other genre-controlled omissions? Examples like (50)-(51), retrieved from

4. While topicalization and focus-movement constructions are not generally categorized as non-declarative constructions, we view them as such here because they represent argumentfocus constructions (Prince 1984). Declaratives, by contrast, are typically associated with predicate-focus (or, equivalently, topic-comment) articulation (Lambrecht 1994: Chapter 4).

5. We do not necessarily share this general assessment. For instance, we think that the made up sentence in (i) could felicitously occur in a diary:

(i) Limbaugh, $\varnothing$ want nothing to do with him.

One might argue that the use of such a sentence in a diary is licensed by general conventions for telegraphic speech in casual conversation and that it therefore need not be treated as an omission licensed by the diary-subject construction. But this then raises the question of how a genre instance is to be defined in a noncircular way: if a first-person omission in a diary occurs in a construction containing a preclausal detached NP, is it not thereby an instance of diary-style omission? 
Google, suggest that Instructional Imperative omission can occur in a predication preceded by a preclausal locative argument: ${ }^{6}$

(50) In a bowl, toss $\varnothing$ with salt and set $\varnothing$ aside.

(51) In a skillet, saute $\varnothing$ until browned but not crisp.

Further, depending on one's analysis of quotative clauses that follow the quoted material, as in (33), above, such examples are a priori evidence of the compatibility of addressee/evaluee omission with fronted constituents. And while (52) is a doctored example, it suggests that match-report omission can occur in a predication featuring a fronted goal argument:

(52) Over the top he headed $\varnothing$ again with only 2 minutes left.

Less equivocal are the binding and control properties of zero arguments in genrebased omissions. For example, omitted subjects of diaries are, in Haegeman's terms, syntactically active: they can bind reflexives (53) and serve as controllers of depictive secondary predicates (54).

(53) Yesterday finally $\emptyset$ managed to get myself back on the end of a paint brush.

(54) Ø Had to stop, wet to skin (Haegeman 1991, (17c))

The omitted arguments of labelese predications can likewise control depictive and resultative secondary predicates, as in (55)-(56), respectively:

(55) $\varnothing$ Stores well at 10C for use throughout the winter.

(56) Ø Biodegrades to carbon dioxide and water.

Similarly, the omitted objects of instructional imperatives can control depictive secondary predicates and bind reflexives, as in (57)-(58):

(57) Avoid chewing $\varnothing$ or swallowing $\varnothing$ whole

(58) Chill dough, then roll Ø to $1 / 4$ "-thick and spread $\varnothing$ with date filling and turn $\varnothing$ over on itself, making a jelly roll.

By contrast, covert objects of match-report predications cannot control secondary predicates denoting current states, as shown by (59). They can, however, control secondary predicates denoting resultant states, as shown by (60), where the PP into the net denotes the location to which the ball is propelled:

(59) He kicked off ${ }^{\star}$ (the ball) still wet.

6. This of course excludes $w h$-fronting, since imperatives are not compatible with questions. 
(60) He smashed $\varnothing$ into the net when a close call went against him.

In sum, the grammatical evidence supports the view that the five omission types are distinct phenomena. Moreover, their grammatical treatment poses various interrelated representational problems. We will address these in the next section.

\section{Constructional analysis}

We propose that genre-based argument omissions are licensed by derivational constructions. In what follows we will describe two of these constructions using conventions of Sign-Based Construction Grammar (SBCG), as proposed by Sag (2007, 2010). SBCG can be regarded as a formalized extension of the Berkeley Construction Grammar (BCG) tradition articulated by Fillmore et al. 1988, Fillmore and Kay 1995, Kay and Fillmore 1999, Kay 2002, Michaelis and Ruppenhofer 2001 and Michaelis and Lambrecht 1996, inter alia. In SBCG, as in BCG, rules of syntactic combination are directly associated with interpretive and use conditions. Proponents of both models assume that grammatical phenomena, rather than being divisible into 'core phenomena' and 'marked' or 'peripheral' phenomena, are arrayed along a continuum of idiomaticity, or generality, of expressions; this continuum is represented by an array of constructions of correspondingly graded generality. Further, BCG and SBCG take a non-modularist perspective on grammar. Rather than seeing syntax, semantics and lexicon as independent modules, with the lexicon characterized as an unstructured set of idiosyncratic form-meaning pairings, the two allied frameworks see a multiple inheritance hierarchy inherent in the lexicon and extend this model to relations among phrasal types. In SBCG, however, generalizations about constructions that were captured by inheritance networks in the BCG tradition are expressed through a hierarchical classification of types. Further, in accordance with Head Driven Phrase Structure Grammar (Pollard and Sag 1994, Sag et al. 2003), from which it derives, and in a departure from the BCG tradition, SBCG presumes that constructions, like the phrase-structure rules of context-free phrase-structure grammar, describe strictly local trees, i.e., mother-daughter configurations. In addition, while in BCG the constructions that regulate the grammatical expression of verbs' arguments (e.g., the Ditransitive and Passive constructions) are represented by schematic lexical entries with which grammatically underspecified verb entries can unify, in SBCG this work is mainly done by a series of unary-branching ('box over box') derivational constructions, which relate two fully specified lexical entries (with the mother representing the 'output' lexeme and the daughter the 'input' lexeme). Of particular relevance for our purposes here is the fact that derivational constructions are used in SBCG to 
remove elements from the valence sets of lexemes in cases of null complementation and to generate lexeme entries that permit such valence reduction.

In SBCG, the basic object of grammatical description is the sign. A language is taken to be an infinite set of signs, and a grammar is taken to be a description of the recursive embedding of signs that constitutes the target language. While the term sign is understood in something close to its Saussurean sense, as a pairing of form and meaning, signs in SBCG are used to model not only words but also phrases. Signs are types of linguistic objects and are organized by means of a type hierarchy (e.g., the sign type word is a subtype of the sign type lexical-sign, as is the sign type lexeme). Formally, a sign is a feature structure that specifies values for the features listed in (61)-(65):

(61) SYN describes the grammatical behavior of a sign. Its values are the features CAT and VAL(ENCE). The values of CAT are complex syntactic categories, represented as typed feature structures, e.g., noun, verb, preposition. The VAL feature represents the objects with which a given sign can combine. The VAL value of pronouns, proper nouns and most common nouns is an empty list. The VAL value of a verb is its combinatoric potential; for example, the VAL value of a transitive verb is $<\mathrm{NP}, \mathrm{NP}>$ while that of a verb phrase, a verb that seeks only a subject, is $\langle\mathrm{NP}\rangle$.

(62) ARG-ST provides a ranked list of a lexical expression's arguments.

(63) SEM describes the meaning of a sign; its values are the features INDEX and FRAMES. INDEX is a constant that represents the extension of a referential sign. The FRAMES feature is used to enumerate the predications that together specify the meaning of a sign. Among the frames that we will consider here are quantifier frames. For example, the meaning of the indefinite article $a$ in English is represented by means of an existentialquantifier frame, whose sole argument is the variable bound by the quantifier in question.

(64) FORM is used to specify the morphological properties of a given sign; the value of FORM is a list of words or morphemes. PHON describes the phonological phrase corresponding to a given sign.

(65) CONTEXT (CNTXT) is used to specify features of context that are relevant to the interpretation and use of a given sign.

Constructions in SBCG are descriptions of the possible signs and combinations of signs (phrases) in the target language; both signs and sign combinations are modeled as feature structures. SBCG recognizes two kinds of constructions: lexical-class constructions, which describe properties common to sets of words and 
lexemes, and combinatory constructions, which describe constructs (Sag 2007, forthcoming). A construct can be viewed as a local tree licensed by a rule of the grammar. Combinatory constructions, which describe possible constructs, are not themselves trees, but are instead feature structures that contain a MOTHER (MTR) feature and a DAUGHTERS (DTRS) feature. Like the phrase-structure rules of context-free grammar, combinatory constructions build phrases (e.g., VP), but they also do some work that phrase-structure rules do not: they build words (e.g., the third-person singular form of the lexeme laugh) and lexemes (e.g., the causative lexeme corresponding to the inchoative lexeme boil). They do this by pairing a mother with one or more daughters. Constructions of the former type are called inflectional constructions and constructions of the latter type are called derivational constructions.

Accordingly, the grammar is viewed as consisting of a lexicon - a finite set of lexical descriptions (descriptions of feature structures whose type is either lexeme or word) - and a set of constructions. We propose (pace Kay, 2004) that argument omission can but need not be licensed by a lexeme. In the case of activity verbs like eat, drink and read, which allow existentially interpreted null-complements in episodic contexts (e.g., Sue ate $\varnothing$ at noon/drank $\varnothing$ at the party/reads $\varnothing$ during breakfast), we concur that the zero argument's potential for null expression is encoded in the lexical entry of the particular verb, as per Kay. An example of a lexical entry including this null complementation option is given in Figure 1.

In Figure 1, we see the lexeme eat, which permits (but does not require) null instantiation of its second argument. Accordingly, the sign types of its valence members are, respectively, overt and (ini). According to the hierarchy of sign types laid out by Kay (2004), this (ini) sign type may resolve to either an overt sign or an ini sign. The latter occurs when the eat lexeme is combined with the Null Complementation construction, a derivational construction that in effect removes an argument from a lexeme's valence set while ensuring that this argument remains

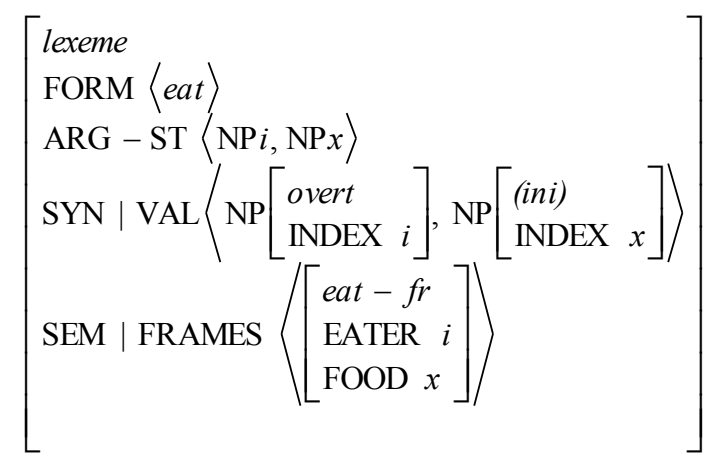

Figure 1. The lexeme eat 
part of the verb's argument-structure set (Kay 2004). A derivational construct licensed by the Null Complementation construction is given in Figure 2.

In the construct shown in Figure 2, the daughter lexeme is identical to that in Figure 1. The MTR lexeme is an alternate drink lexeme which lacks the 'food' argument (the (ini) NP in the daughter's valence set) in its the valence set. The reason that we do not see the ini sign in the valence set of the MTR lexeme is that an ini sign is a type of covert sign; as such, it is subject to the constraint in (66):

(66) covert $\Rightarrow$ sign \& $[$ FORM $<>]$

The constraint in (66) ensures that the type ini has no form value. A sign with no form value will not appear on a lexeme's VAL list, since the VAL value is a list of overt signs. As a subtype of the type null-comp, the ini sign type is also subject to the constraint in Figure 3. The constraint in Figure 3 ensures that if there is a sign of the type null-comp, the lexeme that licenses this sign will have a quantifier frame on its FRAMES list, as does the MTR lexeme in Figure 2. This quantifier frame takes a bound variable (BV) as its argument, and this bound variable shares its

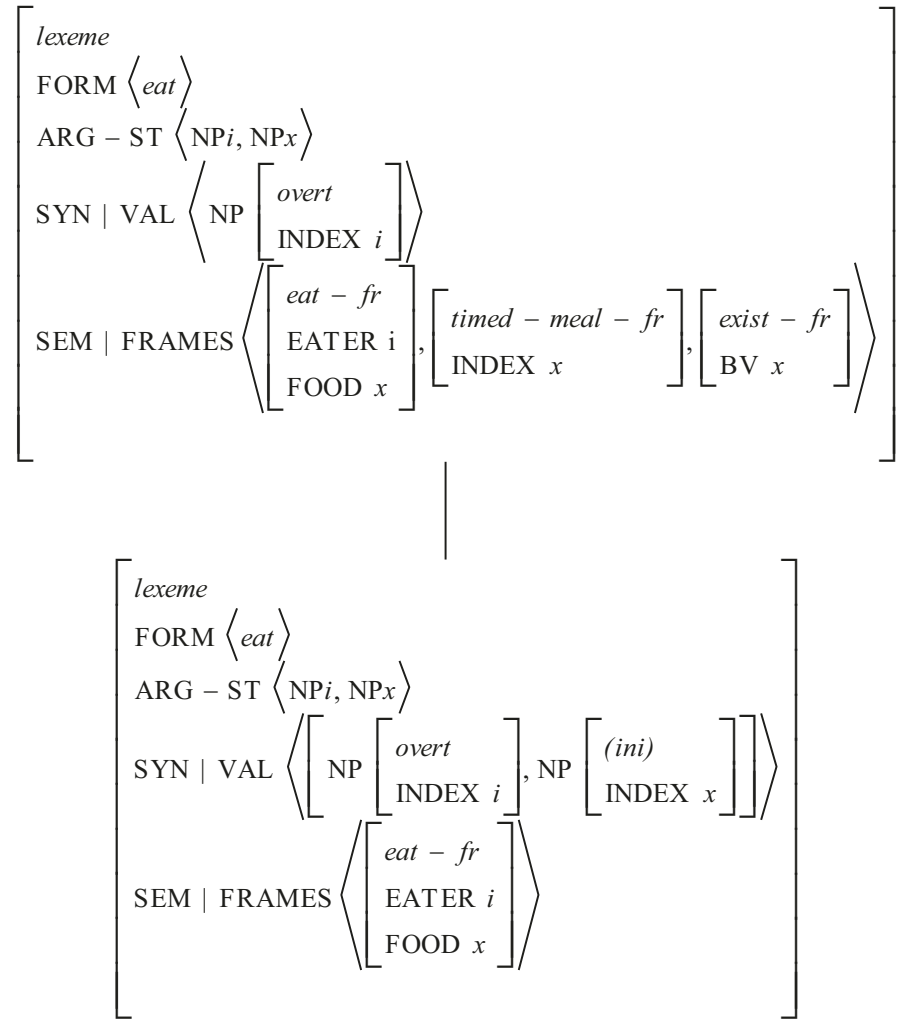

Figure 2. Derivational construct containing eat 


$$
\text { null - comp } \Rightarrow \text { covert \& }\left[\operatorname{SEM}\left[\operatorname{INDEXx}\left\{\left[\begin{array}{c}
\text { frame } \\
\text { BV } x
\end{array}\right]\right\rangle\right]\right]
$$

Figure 3. Null complement type constraint

index with the covert sign. The type constraint in (67) ensures that the quantifier frame is in particular an existential-quantifier frame:

$$
\text { ini } \Rightarrow \text { null-comp \& [SEM [FRAMES <exist-fr }>]]
$$

The constraint in (67) captures the existential interpretation of a missing argument in sentences like (68):

(68) I've eaten.

Sentence (68) means something like 'I've eaten some food at a canonical meal time' rather than 'I've eaten that food at a canonical meal time'.

However, despite what the foregoing discussion has implied, we cannot treat null complementation as a purely lexical affordance. Such an analysis is contraindicated by the facts described in Section 2. For example, as mentioned, verbs like serve do not allow null-instantiated second arguments except in instructional imperatives. The null complementation construction, however, can only 'remove' arguments from the valence sets of verbs like eat and drink, which are 'marked' as permitting null instantiation. Thus, a derivational construction could not be used to account for the omission behavior of a verb like serve, which does not license omission independently of context. While we do not deny that the grammar contains derivational constructions that license constructs like that in Figure 2, we propose two routes to null complementation. First, a lexeme that already contains a valence member of type (ini) or, for that matter, of type (dni) (i.e., an optionally null instantiated argument that receives an anaphoric construal when covert), can unify with the daughter lexeme of a null-instantiation construction, yielding a lexeme with a missing valence member, as in Figure 2. Second, a lexeme that does not already contain an (ini) or ( $d n i)$ valence member can become such a lexeme by derivation, thus enabling it to unify with the daughter lexeme of the Null Complementation construction illustrated in Figure 2. We presume that the derivational constructions which create lexical licensors of null complements, and which thereby 'feed' the Null Complementation construction exemplified in Figure 2 are restricted to certain genres.

Figure 4 shows the Diary Omission construction, the derivational construction that we propose as the licensor of null subject-instantiation in finite predications 


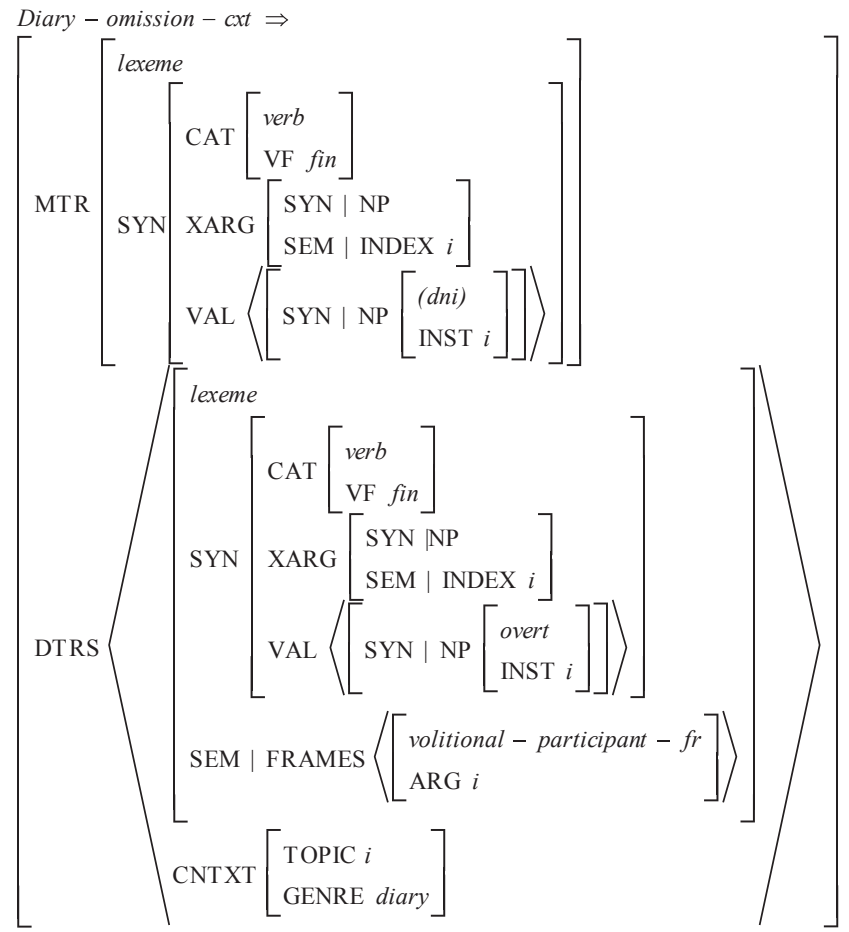

Figure 4. The Diary Omission construction

in the diary genre. The mother of this construction is a finite verbal lexeme that licenses an optionally null instantiated valence member, while the daughter is a finite verbal lexeme that requires this valence member to be overt.

The Diary Omission construction includes the CNTXT feature proposed by Sag $(2007,2010)$ as a method of capturing the use conditions and informationstructure constraints attached to a construction or its arguments. Here we use CNTXT to express two conditions on the daughter lexeme in the construction: the null instantiated argument must be a sentence-level topic (i.e., a predictable argument in the predication) and the construction is conventionally associated with the diary genre. Coindexation is used to indicate that the topical participant is (a) a volitional entity and (b) the external (or highest ranking) argument of the predication, represented by the abbreviation XARG as per SBCG convention. The instantiation type is $(\mathrm{dni})$, indicating that should this lexeme combine with a derivational construction whose daughter matches its type, the MTR lexeme will contain what Kay (2004) refers to as a the-frame. This frame is the anaphoric analog to the exist-frame shown in the MTR lexeme in Figure 2. The the-frame represents the anaphoric interpretation of the null-instantiated argument in the diary genre. 
As we noted earlier, while Haegeman rules out the occurrence of diary-style subject omissions in predications containing preclausal arguments, we may wish to admit such configurations. And in fact, our analysis syntactically allows for diary-subject lexemes to occur in such constructions. That this in fact is a rare occurrence is, in our view, due to pragmatic considerations. Diary style, we postulate, involves what Dowty (1986) calls temporal discourse: a series of declarative predications about a single participant where the reference time of each such predication is construed as later than the prior one. Since interrogatives, topicalization and left dislocation are not part of temporal discourse, the combination of a diary-genre lexeme and one of these constructions creates a pragmatic clash. If our argument is valid, fronting should be rare even in narrative portions of diaries where overt reference is made to the diarist. This prediction appears to be confirmed: the only attested examples of fronting that we have been able to find on various online diary or journalling sites are from non-narrative parts of journal entries, as in (69).

(69) No matter what I do, I don't think I can honestly be happy with how I look these days. In fact, I've never liked how I look. The face I like, but the body needs work.

However, websites like livejournal.com are not necessarily dedicated to the narration of events in the author's personal life, but may instead be focused on particular topics, and frequently also allow for reader comments and feedback. Accordingly, they may constitute separate genres.

Another genre-based omission type that can be viewed as the product of a derivational construction is the instructional imperative. Figure 5 shows the Instructional Imperative construction; it is a subtype of the Imperative construction. Our analysis is based on proposals of Ginzburg and Sag (2000:27-29) and Sag et al. (2003:216-218), who analyze the imperative as a rule that derives a clause (a verb with its subject requirement satisfied) from a base form of the verb containing a second-person NP as its first valence member. By using such a derivational construction to represent instructional imperatives, we can ensure that two arguments, the addressee and the patient, are 'cancelled off' simultaneously from the valence list of the daughter lexeme, a transitive verb. By removing the highest ranking argument from the valence set of the MTR, rather than merely marking this valent as subject to null instantiation, we represent the fact that omission of the addressee (agent) argument is a required feature of imperatives, instructional and otherwise. In instructional imperative constructs, as shown in Figure 5, the MTR is a phrase with an empty valence list: while the verb's (second-person) subject and direct object appear on the valence list of the daughter, both are missing 
from the valence list of the mother. ${ }^{7}$ The two the-frames represent the deictic/ anaphoric interpretation of the null-instantiated addressee $(x)$ and of the undergoer $(y)$. The construction also specifies that any other valents $(L)$ that the daughter may have specified on its valence list must now appear as sisters of the headdaughter on the DTRS list. Via the CNTXT feature instructional imperatives are tied to instructional contexts. Specifically, we assume that our hierarchy of genres contains a node for instructional genres, whose children, among others, include recipes, product labels, and product manuals. Coindexation is used to indicate that the undergoer must be a topic.

In concluding this section, let us return to a puzzle mentioned in the introduction, and consider the solution offered by SBCG. In the introduction, we asked how a genre could be represented by a grammatical construction, since genres are not linguistic objects like words or phrases; rather, they are discourse strategies used to accomplish real-world goals (e.g., giving instructions, narrating a sporting event, recounting one's daily activities). The answer is that constructions do not represent genres. Instead, they license sign types, both phrasal and lexical, that are indexed to genres. Pragmatic constraints on the construal of arguments in a sign's valence set, as well as contextual conditions governing the use of that sign type, create the connection between the genre and the linguistic conventions that define it.

7. An anonymous reviewer asks whether the Instructional Imperative construction could, instead of removing the undergoer argument from the valence list of the DTR lexeme, simply mark that argument as ( $d n i)$ ('optionally null instantiated under an anaphoric interpretation') in the valence of the MTR, as does the Diary Omission construction shown in Figure 4. Our choice of the former solution is the following consideration: the Null Complementation construction, as illustrated by the construct in Figure 2, licenses a relationship between two lexemes, one of which (the MTR lexeme) contains one less valence member than does the DTR lexeme. The Instructional Imperative construction does not, however, contain a lexeme as its MTR; its MTR is instead a clause (a base form of the verb with its subject requirement satisfied). Because it is phrasal, the MTR sign of the Instructional Imperative cannot unify with the (lexeme) DTR sign of the Null Complementation construction. Thus, if we were to propose an Instructional Imperative construction that produced a phrasal MTR sign containing a (dni) undergoer argument in its valence set, we would require a second version of the Null Complementation construction, containing a phrasal MTR, to represent the 'removal' of an imperative predication's undergoer argument in cases where it is covert. Since this solution strikes us as ad hoc, we have chosen to use just one construction, the Instructional Imperative, to 'remove' both actor and undergoer arguments simultaneously. 


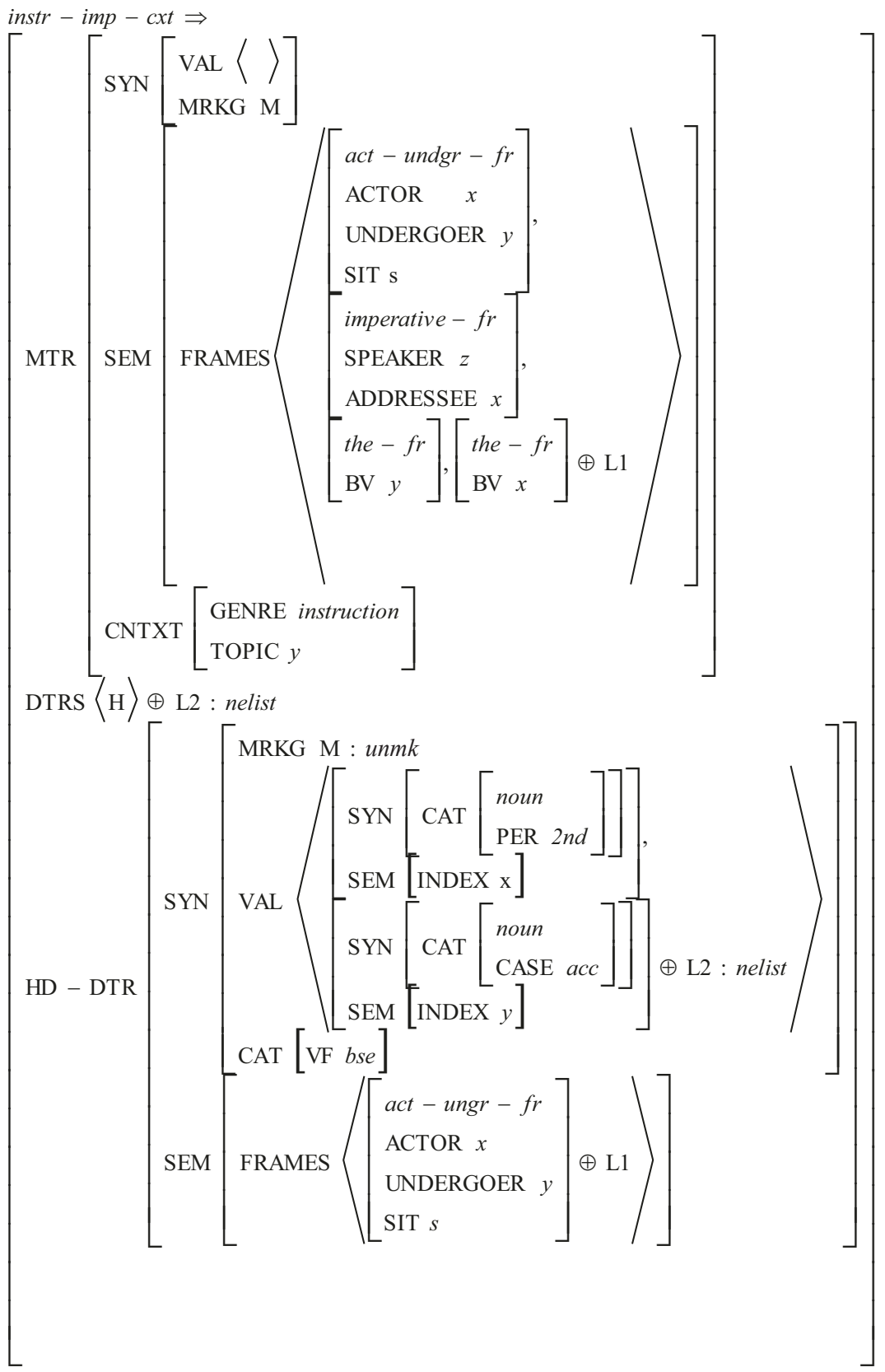

Figure 5. The Instructional Imperative construction 


\section{Conclusion}

Is argument omission lexical, pragmatic or syntactic? The answer that we have given here is 'all three. The argument-omission affordance can be, and typically is, encoded in the valence set of a lexeme like eat or remember, as is the construal type (anaphoric or existential) of each omissible argument. Pragmatic constraints on the use of valence-reducing constructions like the Instructional Imperative construction are represented in the semantic and contextual restrictions that attach to these constructions. The syntactic effects of argument omission are represented by derivational constructions that allow for a mismatch between a verb's combinatoric requirements, as represented by its valence set, and the number of participant roles in its semantic frame.

More specifically, we have suggested that genre-licensed argument omissions are the products of derivational constructions. We believe that construction-based syntax is uniquely well suited for the representation of the syntax-genre interface, because constructions allow us to represent all the patterns along a gradient of schematicity - from detailed lexical-class constructions to highly schematic patterns like the imperative construction. In this respect, construction-based syntax intersects with exemplar-based analysis à la Bybee $(2001,2007)$. Using the mechanism of inheritance, we can acknowledge that the prototypical genre-linked patterns are highly specific, while also recognizing that the specific patterns are instances of the more general ones. For example, the best exemplar of the diary genre is a predication that is declarative, whose null subject is a first-person referent and whose predicate is a finite verb. But of course the prototypical pattern shares pragmatic and formal properties with those clause types whose null subjects are third person but likewise occur in anaphoric chains, as in Section 4.1 above, those clause types whose predicates are nonfinite or even nonverbal, as in, e.g., Feeling fine and And now off to bed, those whose subjects are nonreferential, as in, e.g., Seems I've heard that song before, and even those that have nonfinite predicates, interrogative rather than declarative speech-act function and a second-person null subject, as in e.g., Got milk? In a taxonomically organized grammar, each of these more specific patterns can be shown to be an instance of a more general subject-omission convention for telegraphic speech.

\section{References}

Baker, Collin F., Charles J. Fillmore \& John B. Lowe (1998). The Berkeley FrameNet project. Proceedings of the COLING-ACL. Montreal, Canada. 
Bakhtin, Mikhail (1986). Speech genres and other late essays. Austin, TX: University of Texas Press.

Bell, Anne Olivier (Ed.) (1978-1984). The diary of Virginia Woolf (Vols. I-V). London: Hogarth Press.

Bender, Emily (1999). Constituting context: Null objects in English recipes revisited. Proceedings of the 23rd Annual Penn Linguistics Colloquium.

Biber, Douglas (1995). Dimensions of register variation: A cross-linguistic comparison. Cambridge, UK: Cambridge University Press.

Biber, Douglas, Ulla Connor \& Thomas Albin Upton (2007). Discourse on the move: Using corpus analysis to describe discourse structure. Amsterdam: John Benjamins.

Boas, Hans (2003). A constructional approach to resultatives. Stanford, CA: CSLI.

Burnard, Lou (Ed.) (1995). British National Corpus: Users reference guide for the British National Corpus. Oxford: Oxford University Computing Service.

Bybee, Joan (2007). Frequency of use and the organization of language. Oxford, UK: Oxford University Press.

Bybee, Joan (2001). Phonology and language use. Oxford, UK: Oxford University Press.

Culy, Christopher (1996). Null objects in English recipes. Language Variation and Change, 8.91-124.

Dowty, David (1986). The effects of aspectual class on the interpretation of temporal discourse: Semantics or pragmatics? Linguistics and Philosophy, 9.37-61.

Fillmore, Charles J. (1986). Pragmatically controlled zero anaphora. Proceedings of the Twelfth Annual Meeting of the Berkeley Linguistic Society.

Fillmore, Charles J. \& Paul Kay (1995). Construction Grammar coursebook. Unpublished ms., University of California, Department of Linguistics, Berkeley.

Fillmore, Charles J., Paul Kay \& Mary Catherine O'Connor (1988). Regularity and idiomaticity in grammatical constructions: The case of let alone. Language, 64.501-538.

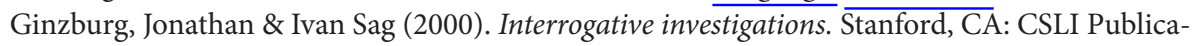
tions.

Goldberg, Adele (2006). Constructions at work: The nature of generalization in language. Oxford, UK: Oxford University Press.

Goldberg, Adele (1995). Constructions: A construction grammar approach to argument structure. Chicago: University of Chicago Press.

Haegeman, Liliane (1987). Register variation in English: Some theoretical observations. Journal of English Linguistics, 20.230-248.

Haegeman, Liliane (1990). Understood subjects in English diaries. On the relevance of theoretical syntax for the study of register variation. Multilingua, 9.157-199.

Kay, Paul (2002). English subjectless tag sentences. Language, $\overline{78.453-481}$.

Kay, Paul (2004). Null instantiation of nominal complements. Unpublished ms., University of California, Department of Linguistics, Berkeley.

Kay, Paul \& Charles J. Fillmore (1999). Grammatical constructions and linguistic generalizations: The 'what's $\mathrm{X}$ doing Y' construction. Language, 75.1-33.

Lambrecht, Knud (1994). Information structure and sentence form. Cambridge: Cambridge University Press.

Lambrecht, Knud \& Laura A. Michaelis (1998). Sentence accent in information questions. Linguistics and Philosophy, 21.477-544.

Michaelis, Laura A. \& Knud Lambrecht (1996). Toward a construction-based model of language function: The case of nominal extraposition. Language, 72.215-247. 
Michaelis, Laura A. \& Josef Ruppenhofer (2001). Beyond alternations: A constructional account of the applicative construction in German. Stanford, CA: CSLI Publications.

Prince, Ellen (1984). Topicalization and left-dislocation: A functional analysis. Annals of the New York Academy of Sciences, 433.213-225.

Ruppenhofer, Josef (2004). The interaction of valence and information structure. Unpublished doctoral dissertation, University of California.

Sag, Ivan (2010). English filler-gap constructions. Language, 86.486-545.

Sag, Ivan (2007). Sign-based construction grammar: An informal synopsis. Unpublished ms., Stanford University, Department of Linguistics, Stanford.

Sag, Ivan, Thomas Wasow, \& Emily Bender (2003). Syntactic theory: A formal introduction. Stanford, CA: CSLI Publications

\section{Author' addresses}

Josef Ruppenhofer

Department of Computational Linguistics

and Phonetics

Saarland University

Building C 7.4

D-66041 Saarbrücken

Germany

josefr@coli.uni-sb.de
Laura A. Michaelis

Department of Linguistics

University of Colorado

295UCB

Boulder, CO 80309

USA

Laura.Michaelis@Colorado.EDU 